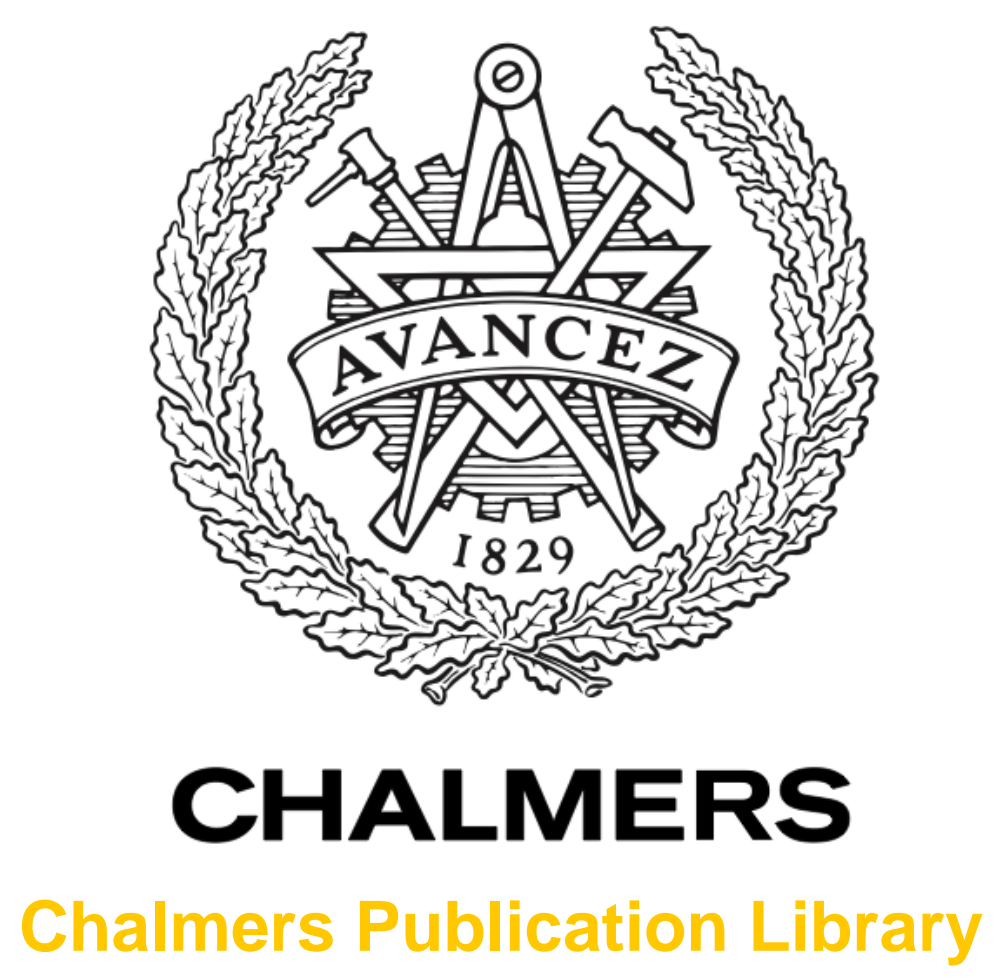

\author{
Large-Scale Analysis of Linear Massive MIMO Precoders in the Presence of Phase \\ Noise
}

This document has been downloaded from Chalmers Publication Library (CPL). It is the author's version of a work that was accepted for publication in:

Large-Scale Analysis of Linear Massive MIMO Precoders in the Presence of Phase Noise

Citation for the published paper:

Krishnan, R. (2015) "Large-Scale Analysis of Linear Massive MIMO Precoders in the

Presence of Phase Noise". Large-Scale Analysis of Linear Massive MIMO Precoders in the

Presence of Phase Noise

Downloaded from: http://publications.lib.chalmers.se/publication/213982

Notice: Changes introduced as a result of publishing processes such as copy-editing and formatting may not be reflected in this document. For a definitive version of this work, please refer to the published source. Please note that access to the published version might require a subscription.

Chalmers Publication Library (CPL) offers the possibility of retrieving research publications produced at Chalmers University of Technology. It covers all types of publications: articles, dissertations, licentiate theses, masters theses, conference papers, reports etc. Since 2006 it is the official tool for Chalmers official publication statistics. To ensure that Chalmers research results are disseminated as widely as possible, an Open Access Policy has been adopted.

The CPL service is administrated and maintained by Chalmers Library. 


\title{
Large-Scale Analysis of Linear Massive MIMO Precoders in the Presence of Phase Noise
}

\author{
R. Krishnan, M. R. Khanzadi, N. Krishnan, Y. Wu, A. Graell i Amat, T. Eriksson, and R. Schober
}

\begin{abstract}
We study the impact of phase noise on the downlink performance of a multi-user multiple-input multiple-output system, where the base station (BS) employs a large number of transmit antennas $M$. We consider a setup where the BS employs $M_{\mathrm{osc}}$ free-running oscillators, and $M / M_{\mathrm{osc}}$ antennas are connected to each oscillator. For this configuration, we analyze the impact of phase noise on the performance of regularized zero-forcing (RZF) precoding, when $M$ and the number of users $K$ are asymptotically large, while the ratio $M / K=\beta$ is fixed. We analytically show that the impact of phase noise on the signal-to-interference-plusnoise ratio (SINR) can be quantified as an effective reduction in the quality of the channel state information available at the BS when compared to a system without phase noise. As a consequence, we observe that as $M_{\mathrm{osc}}$ increases, the SINR of the RZF precoder degrades as the interference power increases, and the desired signal power decreases. On the other hand, the variance of the random phase variations caused by the BS oscillators reduces with increasing $M_{\text {osc }}$. Through simulations, we verify our analytical results, and study the performance of the RZF precoder for different phase noise and channel noise variances.
\end{abstract}

\section{INTRODUCTION}

Massive multiple-input multiple-output (MIMO) is a promising technology for future wireless networks [1]. In particular for downlink transmissions, employing massive antenna arrays at the base station (BS) is expected to provide significant array gains and improved spatial precoding resolution in multi-user (MU) MIMO systems [2]. This in turn is expected to increase the throughput per user equipment (UE), and enable the support of a large number of UEs at the same time. In general, MIMO systems suffer from MU interference during downlink transmission, which is mitigated by means of channel-aware precoding methods implemented at the BS [2]. Capacity-achieving precoding methods, such as dirty-paper coding [2], for the MIMO broadcast channel have high computational complexity. This motivates the need for computationally simpler methods such as linear precoders [3], which have been shown to achieve close-tooptimal performance [3]-[5] when $M$, and the number of UEs, $K$, are asymptotically large.

Implementing linear precoding methods at the BS mandates the availability of reliable channel state information (CSI). In most prior works on MU-MIMO downlink transmission using linear precoders [3]-[5], it is assumed that the hardware components of the MIMO transceiver are ideal. Hardware impairments can affect the CSI quality drastically, and the phase noise caused by noisy local oscillators used in the transceivers is a major contributor to this problem [6]-[8]. As illustrated in [7], [8], phase noise causes partial coherency loss, i.e., the true channel during the data transmission period can become significantly different from the CSI acquired during the training period. This is referred to as the channel-aging phenomenon [9]. It is therefore expected that phase noise at the BS and the UEs will present a serious challenge towards realizing the unprecedented advantages promised by massive MIMO [6]. The effect of phase noise on the uplink performance of a massive MIMO system has been analyzed in [6]-[8]. However, the number of studies on the impact of phase noise on the downlink performance of massive MIMO systems are limited.

In this paper, we analyze the massive MIMO downlink performance of the regularized zero-forcing (RZF) precoder in the presence of oscillator phase noise. We consider a single-cell massive MIMO system comprising one BS serving multiple single-antenna UEs. We analyze a general setup, shown in Fig. 1 , where the BS employs $M_{\text {osc }}$ free-running oscillators, and $M / M_{\text {osc }} \in \mathbb{Z}^{+}$BS antennas are connected to each oscillator. We refer to this as the general oscillator (GO) setup. Two interesting special cases arise from this general setup. In the first case, all BS antennas are connected to a single oscillator (referred to as the common oscillator (CO) setup). In the second case, each BS antenna has its own oscillator (referred to as the distributed oscillator (DO) setup).

For the considered setups, we derive the effective SINR [11] at a given UE for the RZF precoder as $M, K \rightarrow \infty$, while the ratio $M / K=\beta$ is fixed. We show that the impact of phase noise on the SINR of the RZF precoder can be quantified as an effective reduction of the quality of the CSI available at the BS, when compared to the system without phase noise. As a consequence, we observe that as $M_{\text {osc }}$ increases, the SINR degrades. However, the variance of the random phase variations caused by the BS oscillators reduces as $M_{\text {osc }}$ increases. By simulations, we show that the SINR derived is accurate for relevant and practical values of $M$ and $K$. Finally, we compare the achievable rates of the $\mathrm{CO}$ and the DO setups. A general observation for the RZF precoder is that the CO setup performs better than the DO setup when $\beta$ is small. However, the opposite is true when $\beta$ is large and the signal-to-noise ratio (SNR) at the UE is low.

Notation: Vectors and matrices are represented by boldface lower-case and boldface upper-case letters, respectively. The complex Gaussian distribution and the real Gaussian distribution with mean $\mu$ and variance $\sigma^{2}$ are denoted as $\mathcal{C N}\left(\mu, \sigma^{2}\right)$, and $\mathcal{N}\left(\mu, \sigma^{2}\right)$, respectively. The Hermitian, conjugation, transpose, expectation with respect to $\phi$, and trace operators are denoted as $\{\cdot\}^{\mathrm{H}},\{\cdot\}^{*},\{\cdot\}^{\mathrm{T}}, \mathbb{E}_{\phi}\{\cdot\}$, and $\operatorname{tr}\{\cdot\}$, respectively. $\Re\{\cdot\}, \Im\{\cdot\},|\cdot|$ and $\angle$. are the real part, imaginary part, magnitude, and angle of a complex number, respectively. The $\ell 2$ norm of a vector is denoted by $\|\cdot\|$. An $n$-dimensional complex vector is denoted by $\mathbb{C}^{n \times 1}$, while $\mathbb{C}^{n \times m}$ denotes the generalization to an $(n \times m)$ dimensional complex matrix. The integer space is denoted by $\mathbb{Z}$. The diag $\{\ldots\}$ operator generates a diagonal matrix from a given vector, and $\mathbf{I}_{M}$ denotes an $M \times M$ identity matrix.

\section{SySTEM MODEL}

We consider a single-cell system consisting of a BS that serves $K$ UEs. The BS is equipped with $M$ antennas and each UE is equipped with a single antenna. The channel between the 


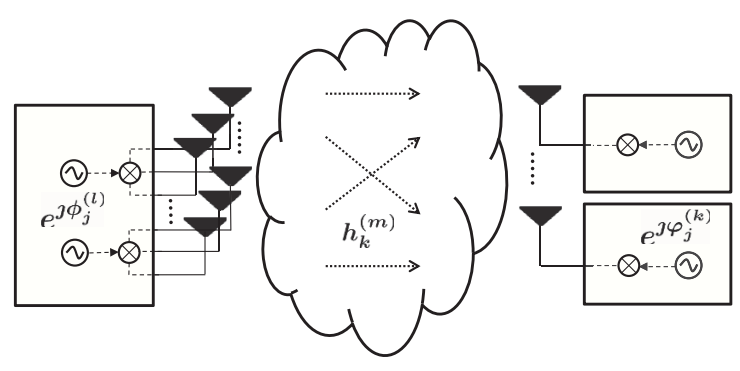

Fig. 1: The general oscillator (GO) setup, where the BS has $M_{\mathrm{osc}}$ free-running oscillators, and $M / M_{\mathrm{osc}} \in \mathbb{Z}^{+} \mathrm{BS}$ antennas are connected to each oscillator.

$m$ th antenna, $m \in\{1, \ldots, M\}$, at the BS and the $k$ th $\mathrm{UE}$, $k \in\{1, \ldots, K\}$, is assumed to be frequency-flat Rayleigh blockfading, and its gain is denoted as $h_{k}^{(m)} \sim \mathcal{C N}\left(0, \sigma_{\mathrm{h}}^{2}\right)$, where $\sigma_{\mathrm{h}}^{2}=1$. Furthermore, $h_{k}^{(m)}$ is the $(k, m)$ th entry of $\mathbf{H} \in \mathbb{C}^{K \times M}$, which represents the channels between the BS and the UEs. The coherence time of the block-fading channel is denoted by $T_{\mathrm{c}}$. Without loss of generality, it is assumed that the large-scale fading component of the channel is unity.

We consider the GO setup where the BS employs $M_{\text {osc }}$ free-running oscillators, and $M / M_{\mathrm{osc}} \in \mathbb{Z}^{+} \mathrm{BS}$ antennas are connected to each oscillator. Considering a discrete-time Wiener phase noise model [10], [15], in the $j$ th symbol interval, the phase noise sample at the $k$ th UE is denoted by $\varphi_{j}^{(k)}$, and that of the $l$ th oscillator at the BS is denoted by $\phi_{j}^{(l)}$, where

$$
\begin{array}{rlrl}
\varphi_{j}^{(k)} & =\varphi_{j-1}^{(k)}+\Delta_{j}^{\varphi}, & \Delta_{j}^{\varphi} & \sim \mathcal{N}\left(0, \sigma_{\varphi}^{2}\right), \\
\phi_{j}^{(l)}=\phi_{j-1}^{(l)}+\Delta_{j}^{\phi}, & \Delta_{j}^{\phi} & \sim \mathcal{N}\left(0, \sigma_{\phi}^{2}\right) .
\end{array}
$$

Here, $k \in\{1, \ldots, K\}, l \in\left\{1, \ldots, M_{\text {osc }}\right\}$, and $\sigma_{\varphi}^{2}$ and $\sigma_{\phi}^{2}$ denote the phase noise increment variances at the UE and the $\mathrm{BS}$, respectively. Since the channel is constant within $T_{\mathrm{c}}$, and given that the $m$ th $\mathrm{BS}$ antenna is connected to the $l$ th oscillator at the $\mathrm{BS}, \theta_{j, k}^{(m)} \triangleq \varphi_{j}^{(k)}+\phi_{j}^{(l)}$ is the phase noise sample that impairs the link between the $k$ th UE and the $m$ th BS antenna.

Time division duplex (TDD) is assumed, where the UEs first transmit time-orthogonal pilots to the BS for channel training. Upon reception of the pilots from the UEs, the BS forms an estimate of the channel between its antennas and the UEs. Exploiting channel reciprocity [1], the channel estimate is then used by the $\mathrm{BS}$ to transmit data in the downlink to the UEs. This training scheme permits a simple channel estimation method at the BS, and as we shall see later, facilitates the large-scale analysis of the MIMO downlink performance [4], [5]. Importantly, this training scheme aids in capturing the channel-aging effect due to phase noise on the SINR achieved at the UEs.

During channel training, the UEs transmit their pilot symbols sequentially in time, meaning that when one UE is transmitting, the other $K-1$ UEs are silent. The pilot signal received at the BS from the $k$ th UE at time instant $j=0$ can be written as

$$
\mathbf{y}_{\mathrm{u}, 0}=\sqrt{p_{\mathrm{u}, k}} \boldsymbol{\Theta}_{0, k} \mathbf{h}_{k} c_{0, k}+\mathbf{w}_{\mathrm{u}, 0}
$$

where $\mathbf{y}_{\mathrm{u}, 0}=\left[y_{\mathrm{u}, 0}^{(1)}, \ldots, y_{\mathrm{u}, 0}^{(M)}\right]^{\mathrm{T}}$, and $y_{\mathrm{u}, 0}^{(m)}$ represents the received signal in the uplink at the $m$ th BS antenna. $p_{\mathrm{u}, k}$ denotes the uplink transmit power of the $k$ th UE, $\boldsymbol{\Theta}_{0, k}=\operatorname{diag}\left\{e^{\jmath \theta_{0, k}^{(1)}} \mathbf{1}_{M / M_{\mathrm{osc}} \times 1}^{\mathrm{T}}, \ldots, e^{\jmath \theta_{0, k}^{\left(M_{\mathrm{osc}}\right)}} \mathbf{1}_{M / M_{\mathrm{osc}} \times 1}^{\mathrm{T}},\right\}$, where $\mathbf{1}_{M / M_{\mathrm{osc}} \times 1}^{\mathrm{T}}$ denotes an all-one vector of length $M / M_{\mathrm{osc}}$, and $\mathbf{h}_{k}=\left[h_{k}^{(1)}, \ldots, h_{k}^{(M)}\right]^{\mathrm{T}} \cdot c_{0, k}$ denotes the pilot symbol transmitted by the $k$ th UE. $\mathbf{w}_{\mathrm{u}, 0}=\left[w_{\mathrm{u}, 0}^{(1)}, \ldots, w_{\mathrm{u}, 0}^{(M)}\right]^{\mathrm{T}}$, where $w_{\mathrm{u}, j}^{(m)} \sim \mathcal{N}\left(0, \sigma_{\mathrm{w}}^{2}\right)$ denotes the zero-mean AWGN random variable (RV) at the $m$ th receive antenna.

Based on $\mathbf{y}_{\mathbf{u}, 0}$, a linear MMSE channel estimate is formed for the $k$ th $\mathrm{UE}$ at time instant $j=0$. This estimate is modeled as [4]

$$
\hat{\mathbf{h}}_{0, k}=\sqrt{q_{0, k}} \boldsymbol{\Theta}_{0, k} \mathbf{h}_{k}+\sqrt{q_{1, k}} \mathbf{w}_{\mathrm{e}, \tau, k} .
$$

Here, $\mathbf{w}_{\mathrm{e}, \tau, k} \in \mathbb{C}^{M \times 1}$ represents the estimation error, and its entries are complex Gaussian independent and identically distributed (i.i.d.) RVs with zero mean and unit variance. $\hat{\mathbf{h}}_{0, k} \in$ $\mathbb{C}^{M \times 1}$ in (4) is the $k$ th row of $\hat{\mathbf{H}}_{0} \in \mathbb{C}^{K \times M}$, which contains the channel estimates of all UE channels. Without loss of generality, we assume that $q_{0}=q_{0, k}$ and $q_{1}=q_{1, k}$, and set the channel estimate variance as $\sigma_{\hat{\mathrm{h}}_{\mathrm{k}}}^{2} \triangleq q_{0} \sigma_{\mathrm{h}}^{2}+q_{1}=1$. The parameter $q_{0} \in[0,1]$ reflects the quality of the channel estimate [4], and is a function of the SNR of the pilot symbol transmission in (4).

Let the data transmission on the downlink from the BS to the UEs commence in the symbol interval $j=\tau, \tau<T_{\mathrm{c}} . \tau$ denotes the symbol periods that have elapsed since the uplink transmission of the pilot symbols from the $k$ th UE. Even though the channel remains a constant during this elapsed time period, the phase noise caused by the oscillators at the BS and the UE drifts randomly. The signal received by the $k$ th $\mathrm{UE}$ at time instant $\tau$ is written as

$$
\begin{aligned}
y_{\mathrm{d}, \tau}^{k} & =\mathbf{h}_{\tau, k}^{\mathrm{T}} \boldsymbol{\Theta}_{\tau, k} \mathbf{x}_{\tau}+w_{\mathrm{d}, \tau, k} \\
& =\mathbf{h}_{\tau, k}^{\mathrm{T}} \boldsymbol{\Theta}_{\tau, k} \sum_{k_{1}=1}^{K} \mathbf{g}_{0, k_{1}} c_{\tau, k_{1}}+w_{\mathrm{d}, \tau, k} .
\end{aligned}
$$

In (5), $\mathbf{x}_{\tau} \in \mathbb{C}^{M \times 1}$ is the transmit signal, and in (6), $\mathbf{x}_{\tau}$ is written as a linear combination of the data symbols $c_{\tau, k_{1}}, k_{1} \in$ $\{1, \ldots, K\}$, transmitted to the $K$ UEs. The data symbols are assumed to be circularly symmetric, but not necessarily Gaussian distributed [15]. $\mathbf{g}_{0, k} \in \mathbb{C}^{M \times 1}$ is the $k$ th column of the downlink precoding matrix $\mathbf{G}_{0} \in \mathbb{C}^{M \times K}$, where $\mathbf{G}_{0}=\left[\mathbf{g}_{0,1}, \ldots, \mathbf{g}_{0, K}\right]$. $w_{\mathrm{d}, \tau, k} \sim \mathcal{C N}\left(0, \sigma_{\mathrm{w}}^{2}\right)$ denotes the AWGN RV at the $k$ th UE. Furthermore, $\boldsymbol{\Theta}_{\tau, k}$ represents the random phase noise variations caused by the noisy oscillators at the BS and the UE during the data transmission phase.

We consider RZF precoding in (6), i.e., $\mathbf{G}_{0}$ is written as [4]

$$
\mathbf{G}_{0}=\xi\left(\hat{\mathbf{H}}_{0}^{\mathrm{H}} \hat{\mathbf{H}}_{0}+M \alpha \mathbf{I}_{M}\right)^{-1} \hat{\mathbf{H}}_{0}^{\mathrm{H}} \mathbf{P}^{\frac{1}{2}}
$$

where $\mathbf{P} \triangleq \operatorname{diag}\left\{p_{1}, \ldots, p_{K}\right\}, p_{k}$ denotes the power allocated to the $k$ th UE, and the normalization parameter $\xi$ is set such that the precoder satisfies the power constraint $\operatorname{tr}\left(\mathbf{G}_{0}^{\mathrm{H}} \mathbf{G}_{0}\right)=1$.

\section{LARGE-ScAle AnAlysis of the ReCEIVED Signal AND ACHIEvable RATES}

In this section, we use tools from random matrix theory [14] to analyze the received signal model in (6). Specifically, we present a simplification of the desired signal term in (6) for the GO setup when $M, K \rightarrow \infty$, while $M / K=\beta$. Notably, we will show that in the $\mathrm{CO}$ and DO setups, the multiple-input single-output (MISO) system model in (6) can be re-written as an equivalent single-input single-output (SISO) phase noise channel including the effects of phase noise, AWGN, and interference [15]. Furthermore, we define the effective SINR, and discuss the achievable rates for the GO setup. 


\section{A. Received Signal Model}

For the RZF precoder in (7), the received signal at the $k$ th UE in (6) becomes

$$
\begin{aligned}
y_{\mathrm{d}, \tau}^{k}= & \mathbf{h}_{k}^{\mathrm{T}} \boldsymbol{\Theta}_{\tau, k} \mathbf{G}_{0} \mathbf{c}_{\tau}+w_{\mathrm{d}, \tau, k} \\
= & \underbrace{\sqrt{p_{k}} \mathbf{h}_{k}^{\mathrm{T}} \boldsymbol{\Theta}_{\tau, k} \xi\left(\hat{\mathbf{H}}_{0}^{\mathrm{H}} \hat{\mathbf{H}}_{0}+M \alpha \mathbf{I}_{M}\right)^{-1} \hat{\mathbf{h}}_{0, k}^{*}}_{\triangleq \mathrm{I}_{\mathrm{sig}}} c_{\tau, k} \\
& +\underbrace{\mathbf{h}_{k}^{\mathrm{T}} \boldsymbol{\Theta}_{\tau, k} \xi\left(\hat{\mathbf{H}}_{0}^{\mathrm{H}} \hat{\mathbf{H}}_{0}+M \alpha \mathbf{I}_{M}\right)^{-1} \hat{\mathbf{H}}_{0,-k}^{\mathrm{H}} \mathbf{P}_{-k}^{\frac{1}{2}}}_{\triangleq \mathbf{I}_{\mathrm{int}}} \mathbf{c}_{\tau,-k} \\
& +w_{\mathrm{d}, \tau, k} .
\end{aligned}
$$

In (8), we have introduced the following definitions: $\hat{\mathbf{H}}_{0,-k}=\left[\hat{\mathbf{h}}_{0,1}, \ldots, \hat{\mathbf{h}}_{0, k-1}, \hat{\mathbf{h}}_{0, k+1}, \ldots, \hat{\mathbf{h}}_{0, K}\right], \quad \mathbf{P}_{-k}=$ $\operatorname{diag}\left\{p_{1}, \ldots, p_{k-1}, p_{k+1}, \ldots, p_{K}\right\}, \quad$ and $\quad \mathbf{c}_{\tau,-k}=$ $\left[c_{\tau, 1}, \ldots, c_{\tau, k-1}, c_{\tau, k+1}, \ldots, c_{\tau, K}\right]^{\mathrm{T}}$. Furthermore, $\mathrm{I}_{\text {sig }} \in \mathbb{C}$ and $\mathbf{I}_{\text {int }}^{\mathrm{T}} \in \mathbb{C}^{M-1 \times 1}$ denote the scaling factors associated with the desired symbol and the interfering symbols at the $k$ th UE, respectively. The factor $I_{\text {sig }}$ is simplified in the following proposition.

Proposition 1: Consider an RZF precoded downlink transmission from a BS having $M$ antennas to $K$ single-antenna UEs employing TDD in the presence of oscillator phase noise. Let $\alpha>0, M / K=\beta, \beta \geq 1$, and $q_{0} \in[0,1]$. Assume that $\frac{1}{M} \hat{\mathbf{H}}^{\mathrm{H}} \hat{\mathbf{H}}$ has uniformly bounded spectral norm for all $M$. Then, the desired signal factor $\mathrm{I}_{\text {sig }}$, for $M, K \rightarrow \infty$, can be simplified to

$$
\mathrm{I}_{\mathrm{sig}}=\sqrt{p_{k} q_{0}} T_{\mathrm{PN}} \xi t e^{\jmath\left(\varphi_{\tau}^{(k)}-\varphi_{0}^{(k)}\right)},
$$

where

$$
\begin{aligned}
T_{\mathrm{PN}} & \triangleq \lim _{M \rightarrow \infty} \frac{1}{M} \operatorname{tr}\left\{\Delta \boldsymbol{\Phi}_{\tau}\right\} \\
\xi & =\lim _{M, K \rightarrow \infty} \sqrt{\frac{M(1+m(-\alpha))^{2}}{m^{\prime}(-\alpha) \sum_{k=1}^{K} p_{k}}} \\
m(-\alpha) & =\frac{\beta-1-\alpha \beta+\sqrt{\beta^{2} \alpha^{2}+2(\beta+1) \alpha \beta+(1-\beta)^{2}}}{2 \alpha \beta} \\
t & =\frac{m(-\alpha)}{m(-\alpha)+1} .
\end{aligned}
$$

In $(10), \Delta \boldsymbol{\Phi}_{\tau}=\operatorname{diag}\left\{e^{\jmath\left(\phi_{\tau}^{(1)}-\phi_{0}^{(1)}\right)} \mathbf{1}_{M / M_{\mathrm{osc}} \times 1}^{\mathrm{T}}, \ldots\right.$, $\left.e^{\jmath\left(\phi_{\tau}^{\left(M_{\mathrm{osc}}\right)}-\phi_{0}^{\left(M_{\mathrm{osc}}\right)}\right)} \mathbf{1}_{M / M_{\mathrm{osc}} \times 1}^{\mathrm{T}}\right\}, m(-\alpha)$ in (12) is the Stieltjes Transform of the Marchenko-Pastur Law [14, Eqs. (1.12, 2.43)], and $m^{\prime}(-\alpha)=\left.\frac{d m(z)}{d z}\right|_{z=-\alpha}$.

Proof: Please refer to Theorem 1 for a discussion on the proof, and to [15, Proposition 1] for a detailed proof.

Remark 1: The terms $t$ and $\xi$ in (9) depend on $\alpha$ and $\beta$, and capture the channel hardening effect [1], [4] that results from the averaging of the random fading channels when RZF precoding is used, and $M, K \rightarrow \infty$. The term $T_{\mathrm{PN}}$ in (10) captures the effects of phase noise variations at the BS between the training and the data transmission phases, and is given by

$$
T_{\mathrm{PN}}=\frac{1}{M_{\mathrm{osc}}} \sum_{l=1}^{M_{\mathrm{osc}}} e^{\jmath\left(\phi_{\tau}^{(l)}-\phi_{0}^{(l)}\right)} .
$$

Specifically, for the CO setup, where $\Delta \boldsymbol{\Phi}_{\tau}=$ $e^{\jmath\left(\phi_{\tau}-\phi_{0}\right)} \mathbf{I}_{M}$, and the DO setup, where $\Delta \boldsymbol{\Phi}_{\tau}=$

$$
\begin{gathered}
\operatorname{diag}\left\{e^{\jmath\left(\phi_{\tau}^{(1)}-\phi_{0}^{(1)}\right)}, \ldots, e^{\jmath\left(\phi_{\tau}^{(M)}-\phi_{0}^{(M)}\right)}\right\}, \text { (14) reduces to [7] } \\
T_{\mathrm{PN}}=\left\{\begin{array}{ll}
e^{\jmath\left(\phi_{\tau}-\phi_{0}\right)} & \text { CO setup } \\
e^{-\frac{\tau \sigma_{\phi}^{2}}{2}} & \text { DO setup }
\end{array} .\right.
\end{gathered}
$$

Inspection of $T_{\mathrm{PN}}$ in (14) and (15) reveals that $\angle T_{\mathrm{PN}}$ reflects the random phase variations caused by the oscillators at the BS. The variance of $\angle T_{\mathrm{PN}}$ decreases as $M_{\mathrm{osc}}$ increases, while its mean is zero for all values of $M_{\mathrm{osc}}$. $\left|T_{\mathrm{PN}}\right|$ represents the random amplitude variations in $I_{\text {sig }}$ caused by transmissions using distributed (asynchronous) oscillators at the BS. As $M_{\text {osc }}$ increases, the mean of $\left|T_{\mathrm{PN}}\right|$ reduces from 1 to $\exp \left(-\frac{\tau \sigma_{\phi}^{2}}{2}\right)$. Specifically, for the CO setup, $\left|T_{\mathrm{PN}}\right|=1$, while for the DO setup, $\left|T_{\mathrm{PN}}\right|=\exp \left(-\frac{\tau \sigma_{\phi}^{2}}{2}\right)$. The variance of $\left|T_{\mathrm{PN}}\right|$ is maximal for $M_{\mathrm{osc}}=2$, and decreases as $M_{\mathrm{osc}}$ increases. In summary, when $2 \leq M_{\mathrm{osc}}<\infty$, there are random variations in $T_{\mathrm{PN}}$. In the DO setup ${ }^{1}$, where $M_{\mathrm{osc}}=M, T_{\mathrm{PN}}$ hardens to a deterministic value that depends on $\tau$ and $\sigma_{\phi}^{2}$. However, this hardening effect is not observed in the CO setup as the phase noise caused by the $\mathrm{BS}$ oscillator does not average out.

From the central limit theorem (as $K \rightarrow \infty$ ), and since the symbols $\mathbf{c}_{\tau}$ are circularly symmetric, the interference term $\mathbf{I}_{\text {int }} \mathbf{c}_{\tau,-k}$ in (8) is a circularly symmetric Gaussian RV for both the CO and the DO setups. Furthermore, this term is uncorrelated (and hence independent) from the signal term. In the case where $2 \leq M_{\text {osc }}<\infty, \mathbf{I}_{\text {int }} \mathbf{c}_{\tau--k}$ is non-Gaussian, but still uncorrelated from the signal term. Further analysis of $\mathbf{I}_{\text {int }} \mathbf{c}_{\tau,-k}$ can be found in [15]. Upon applying (9), (15) in (8), we have

$$
y_{\mathrm{d}, \tau}^{k}=\sqrt{p_{k} q_{0}} T_{\mathrm{PN}} \xi t e^{\jmath\left(\varphi_{\tau}^{(k)}-\varphi_{0}^{(k)}\right)} c_{\tau, k}+\mathbf{I}_{\mathrm{int}} \mathbf{c}_{\tau,-k}+w_{\mathrm{d}, \tau, k} .
$$

For the CO and the DO setups, the MISO system model in (6) and (8) becomes an equivalent SISO phase noise channel in (16) [15]. However, when $2 \leq M_{\text {osc }}<\infty$, (16) still corresponds to a MISO phase noise channel, since $T_{\mathrm{PN}}$ in (14) depends on the random phase noise variations of the multiple oscillators at the BS.

\section{B. Effective SINR and Achievable Rates}

For the CO and the DO setups, we define the effective SINR based on the SISO phase noise channel in (16) as [15]

$$
\operatorname{SINR}_{k}=\frac{\left|\mathrm{I}_{\mathrm{sig}}\right|^{2}}{\left\|\mathbf{I}_{\mathrm{int}}\right\|^{2}+\sigma_{\mathrm{w}}^{2}} .
$$

Since the phase noise in (2) drifts symbol-by-symbol, the SINR, which depends on $\tau$ (8), also varies symbol-by-symbol. In order to analyze the achievable rate of a given UE based on the SINR in (17), we model the phase noise to be a constant within a block of symbols [15]. Therefore, the SINR which is computed for a given $\tau$ corresponds to the SINR associated with a block of symbols, and can be used to determine the achievable rate [12]. Note that this model is implicity used in [6]-[8]. Furthermore, this model is only used to evaluate the achievable rates based on the SINR derived, and is not required, per se, for deriving the SINR and the other analytical results in this paper. The achievable rate computed based on this model is an upperbound for the case where the SINR varies symbol-by-symbol.

\footnotetext{
${ }^{1}$ It is important to note that the results for the DO setup also hold in the case where $M_{\mathrm{osc}} \rightarrow \infty$, while the ratio $M / M_{\mathrm{osc}}$ is fixed.
} 
Based on the effective SINR in (17), an upper bound for the achievable rate of the $k$ th UE for the CO and the DO setups for a given block of symbols (i.e., given $\tau$ ) is [15], [16]

$$
C\left(\mathrm{SINR}_{k}\right) \leq \log _{2}\left(1+\mathrm{SINR}_{k}\right) .
$$

This upper bound, which corresponds to the AWGN channel capacity, is generally tight for low-to-medium SINR values. Another upper bound for the achievable rate for the $\mathrm{CO}$ and the DO setups, which is generally tight at high SINR, was derived by Lapidoth et al. [13], and is given by

$C\left(\operatorname{SINR}_{k}\right) \leq \frac{1}{2} \log _{2}\left(4 \pi^{2} \operatorname{SINR}_{k}\right)-\frac{1}{2} \log _{2}\left(2 \pi e \tau\left(\sigma_{\varphi}^{2}+\delta_{\mathrm{pn}} \sigma_{\phi}^{2}\right)\right)$,

where $\delta_{\mathrm{pn}}=1$, when $M_{\mathrm{osc}}=1$, and $\delta_{\mathrm{pn}}=0$, otherwise. In (19), the second term represents the differential entropy of the phase noise process, $\varphi_{\tau}^{(k)}-\varphi_{0}^{(k)}+\delta_{\mathrm{pn}}\left(\phi_{\tau}-\phi_{0}\right)$. The result in (19) holds under the assumption that the phase-noise process is stationary, and has a finite differential-entropy rate. Combing (18) and (19), the achievable rate for the CO and the DO setups can be tightly upper-bounded as $C\left(\mathrm{SINR}_{k}\right) \leq$ $\min \{$ Rate in (18), Rate in (19)\} [15].

There are no results available for the achievable rates for the MISO phase noise channel in (8), when $2 \leq M_{\text {osc }}<\infty$. However, the randomness of $T_{\mathrm{PN}}$ in this case is reminiscent of fading channels. Assuming ergodicity for the effective channel in (16), the achievable rate is written as [11, Lemma 1]

$$
\begin{aligned}
C\left(\operatorname{SINR}_{k}\right) & =\mathbb{E}_{\phi} \log _{2}\left(1+\frac{\left|\mathrm{I}_{\mathrm{sig}}\right|^{2}}{\left\|\mathbf{I}_{\mathrm{int}}\right\|^{2}+\sigma_{\mathrm{w}}^{2}}\right) \\
& \approx \log _{2}\left(1+\frac{\mathbb{E}_{\phi}\left|\mathrm{I}_{\mathrm{sig}}\right|^{2}}{\mathbb{E}_{\phi}\left\|\mathbf{I}_{\mathrm{int}}\right\|^{2}+\sigma_{\mathrm{w}}^{2}}\right),
\end{aligned}
$$

where $\mathbb{E}_{\phi}$ denotes the expectation operation with respect to the phase noise at the BS. The accuracy of this approximation increases with increasing $M_{\mathrm{osc}}$. Also, $\mathrm{I}_{\mathrm{sig}}$ and $\mathbf{I}_{\text {int }}$ are not required to be independent. This motivates the definition of an effective SINR for $2 \leq M_{\mathrm{osc}}<\infty$ as

$$
\operatorname{SINR}_{k}=\frac{\mathbb{E}_{\phi}\left|\mathrm{I}_{\mathrm{sig}}\right|^{2}}{\mathbb{E}_{\phi}\left\|\mathbf{I}_{\mathrm{int}}\right\|^{2}+\sigma_{\mathrm{w}}^{2}} .
$$

The effective SINR in (22) reduces to (17) for the CO and the DO setups. Also, note that the achievable rate computed in (21) only depends for the SINR achieved at the UE, and does not account for the reduced variance of the phase noise variations at the BS when $M_{\mathrm{osc}}>1$. However, the rate in (21) is a tight upper bound for low-to-medium SINR values.

\section{SINR ANALYSIS}

In this section, we introduce Theorem 1 , which provides the analytical form for the effective SINR achievable at a given UE for the RZF precoder.

Theorem 1: Consider an RZF precoded downlink transmission from a BS having $M$ antennas to $K$ single-antenna UEs employing TDD in the presence of oscillator phase noise. Let $\alpha>0$, $\beta \geq 1, q_{0} \in[0,1]$, and $\mathrm{SINR}_{k}$ denote the effective SINR at the $k$ th UE. Then,

$$
\mathrm{SINR}_{k}-\mathrm{SINR}_{\mathrm{rzf}_{k}} \stackrel{M, K \rightarrow \infty}{\longrightarrow} 0
$$

almost surely, and the effective SINR associated with the $k$ th UE for the GO setup is given as

$$
\operatorname{SINR}_{\mathrm{rzf}_{k}}=\frac{p_{k} t^{2} q_{0} \mathbb{E}_{\phi}\left|T_{\mathrm{PN}}\right|^{2}}{\frac{t_{2}}{M}\left(1-t q_{0} \mathbb{E}_{\phi}\left|T_{\mathrm{PN}}\right|^{2}-\frac{t q_{0} \mathbb{E}_{\phi}\left|T_{\mathrm{PN}}\right|^{2}}{(1+m(-\alpha))}\right)+\frac{\sigma_{\mathrm{w}}^{2}}{\xi^{2}}}(24)
$$

with

$$
\begin{aligned}
& \mathbb{E}_{\phi}\left|T_{\mathrm{PN}}\right|^{2} \triangleq \mathbb{E}_{\phi}\left|\frac{1}{M} \operatorname{tr}\left\{\Delta \boldsymbol{\Phi}_{\tau}\right\}\right|^{2}=\frac{1-e^{-\tau \sigma_{\phi}^{2}}}{M_{\mathrm{osc}}}+e^{-\tau \sigma_{\phi}^{2}}(25) \\
& t_{2}=\sum_{\substack{k_{1}=1, k_{1} \neq k}}^{K} p_{k_{1}} \frac{m^{\prime}(-\alpha)}{(1+m(-\alpha))^{2}}
\end{aligned}
$$

where $t, m(-\alpha), \xi$, and $T_{\mathrm{PN}}$ are as given in (10)-(13). Specifically, $\mathbb{E}_{\phi}\left|T_{\mathrm{PN}}\right|^{2}=\exp \left(-\tau \sigma_{\phi}^{2}\right)$ for the DO setup, and $\mathbb{E}_{\phi}\left|T_{\mathrm{PN}}\right|^{2}=1$ for the $\mathrm{CO}$ setup.

Proof: For brevity, we present only a sketch of the proof here. First, we derive $\xi$, such the RZF precoder $\mathbf{G}_{0}$ in (7) satisfies the power constraint $\operatorname{tr}\left(\mathbf{G}_{0}^{\mathrm{H}} \mathbf{G}_{0}\right)=1$. Then, we derive the signal term $I_{\text {sig }}$, and the interference power $\left\|\mathbf{I}_{\mathrm{int}}\right\|^{2}$ using tools from random matrix theory [4], [14]. Also, when deriving the signal term $I_{\text {sig }}$, we prove Proposition 1. For the complete proof, please refer to [15, Theorem 1]

Remark 2: Theorem 1 captures the effect of phase noise on the SINR as an additional penalty to the quality of the channel estimate-when phase noise is present, the quality of the channel estimate, $q_{0}$, degrades to $q_{0} \mathbb{E}_{\phi}\left|T_{\mathrm{PN}}\right|^{2}$ in the GO setup (25). The quality of the effective channel estimate decreases as $M_{\text {osc }}$ increases (25). Also, the effective quality is reduced when $\tau$ or $\sigma_{\phi}^{2}$ increase. In the DO setup, the quality of the channel estimate diminishes by a factor of $\exp \left(-\tau \sigma_{\phi}^{2}\right)$. However, in the $\mathrm{CO}$ setup, there is no reduction in the quality of the channel estimate due to noisy oscillators at the BS or the UEs as $\left|T_{\mathrm{PN}}\right|^{2}=1$.

Clearly, the effect of channel aging on the SINR increases as $M_{\text {osc }}$ increases-the SINR in (24) decreases as $M_{\text {osc }}$ increases. This results from the degradation of the desired signal power by a factor $\mathbb{E}_{\phi}\left|T_{\mathrm{PN}}\right|^{2}<1$ when $M_{\mathrm{osc}} \geq 2$, implying that the desired signal power decreases as $M_{\text {osc }}$ increases. On the other hand, the MU interference power increases with $M_{\mathrm{osc}}$, as can be seen in (24). This is because the CSI quality deteriorates due to phase noise (25), thereby reducing the interference suppression capability of the RZF precoder. Specifically, for the CO setup, there is no effect of phase noise on the SINR since the desired signal power and the MU interference power are identical to the case when only AWGN is present.

\section{Simulation Results}

In this section, the analytical results for the RZF precoder presented in Section IV are verified by comparing them against the results obtained from MC simulations. Even though the analytical results are derived for $M, K \rightarrow \infty$, in the sequel, we observe that these results concur with those from simulations for finite values of $M$ and $K$.

We simulate the system model specified in (6) using the RZF precoder, and numerically evaluate the effective SINR in (22). Then, the achievable rate in the downlink for a given UE is computed using (21) for all values of $M_{\mathrm{osc}}$, unless otherwise stated. Recall that this evaluation only depends on the SINR achieved at the UE, and does not account for the reduced variance 


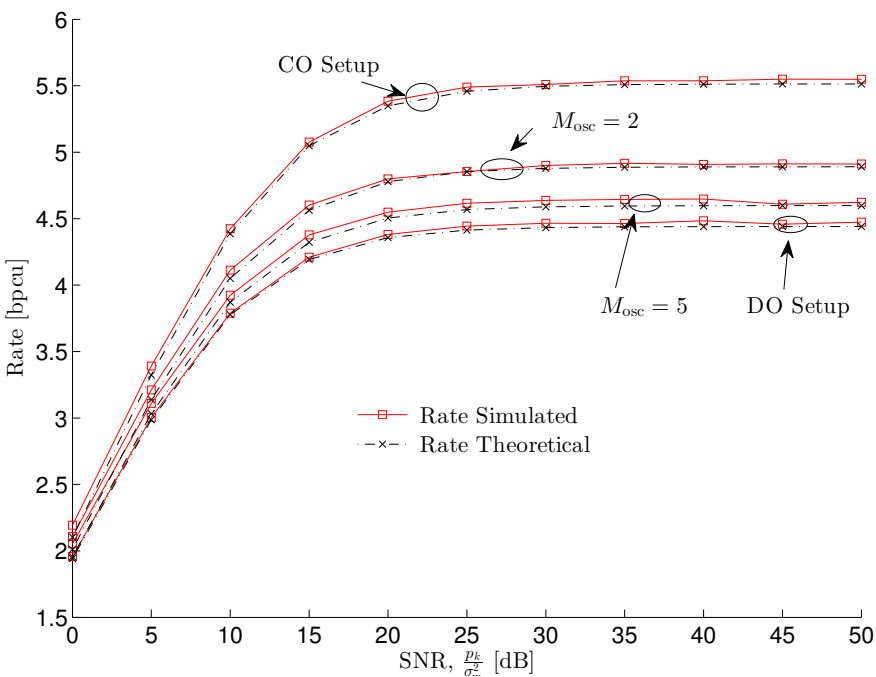

Fig. 2: $C\left(\mathrm{SINR}_{k}\right)$ for the optimized RZF precoder for $\beta=5, M=50, \sigma_{\phi}=$ $\sigma_{\varphi}=6^{\circ}$, and $q_{0}=0.9$.

of the phase noise variations at the BS when $M_{\mathrm{osc}}>1$. We will evaluate the achievable rates for the $\mathrm{CO}$ and the DO setups as $C\left(\mathrm{SINR}_{k}\right)=\min \{$ Rate in (18), Rate in (19) $\}$ [15] when exclusively comparing their performances in Fig. 4. Thereby, the effects of both SINR and the differential entropy rates on the achievable rates of the $\mathrm{CO}$ and the DO setups are taken into account.

The system considered consists of a single cell with a BS having $M=50$ antennas. Setting $\beta=5$, the number of UEs served by the BS is $K=10$. The channel between a BS antenna and a UE is drawn from an i.i.d. complex Gaussian distribution, i.e., $h_{k}^{(m)} \sim \mathcal{C N}(0,1)$. The coherence time of the channel is set to $T_{\mathrm{c}}=100$ data symbol periods, thus resulting in an i.i.d. Rayleigh block-fading channel. The Monte-Carlo (MC) simulations are conducted for 10000 independent channel realizations. The phase noise is simulated as a discrete Wiener process (1), with increment standard deviation $\sigma_{\varphi}=\sigma_{\phi}=6^{\circ}$ [15]. Unless otherwise stated, the time elapsed between the training period of the $k$ th $\mathrm{UE}$ and the data transmission period is set to $\tau=K=10$ symbol periods. Furthermore, all UEs use the same training power, and for downlink transmission, equal power is allocated by the BS to all UEs, i.e., $\mathbf{P}=\frac{1}{K} \mathbf{I}_{K}$. The quality of the channel estimate is set to $q_{0}=0.9$ [4]. This is reasonable given that the UEs can choose to transmit at power levels $p_{\mathrm{u}, k}$ such that the desired $q_{0}$ is attained.

\section{A. Verification of the SINR Result in Theorem 1}

Fig. 2 compares the rate achieved with RZF precoded transmission from the BS to the UEs for different SNR values, where the SNR at the $k$ th UE is defined as $\frac{p_{k}}{\sigma^{2}}$. The value of $\alpha$ used in this simulation is evaluated as in [15, Eq. 35]. We see that the achievable rate (21) for the RZF precoder based on the effective SINR in (24) concurs with the rate achieved in simulations for all the values of $M_{\text {osc }}$ considered. Furthermore, the SINR of the RZF precoder decreases with increasing $M_{\mathrm{osc}}$. Therefore, in terms of SINR degradation due to channel aging caused by phase noise, the CO setup is more robust than the GO setup when $M_{\mathrm{osc}} \geq 2$. Also, the performance of the GO setup for $M_{\mathrm{osc}}=5$ is close to that of the DO setup.

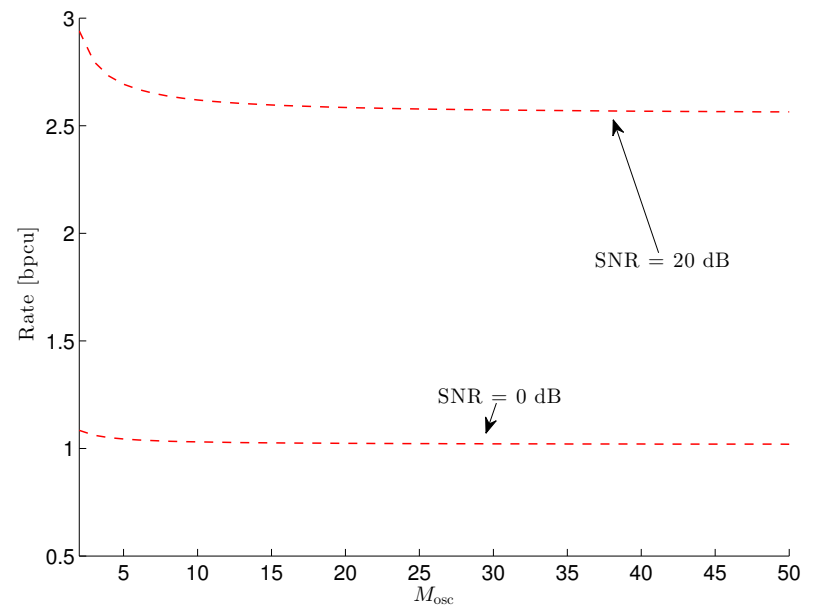

Fig. 3: $C\left(\mathrm{SINR}_{k}\right)$ for the RZF precoder in the GO setup, where $1 \leq M_{\mathrm{osc}} \leq$ $M, M / M_{\text {osc }} \in \mathbb{Z}^{+}$, for $M=50, \beta=2, q_{0}=0.9, \sigma_{\phi}=\sigma_{\varphi}=0.06^{\circ}$, $\tau=T_{\mathrm{C}}=0.25 \mathrm{~ms}$ (10 ${ }^{4}$ symbol periods) [17], and different SNR values.

\section{B. Example Based on LTE Specifications}

We analyze an example based on long-term evolution (LTE) system specifications [17], where we account for practical values of $T_{\mathrm{c}}$, symbol time $T_{\mathrm{s}}$, bandwidth $B W, \tau$, center frequency $f_{\mathrm{c}}$, doppler spread $f_{\mathrm{d}}, \sigma_{\phi}$, and $\sigma_{\varphi}$. We choose $T_{\mathrm{s}}=0.032 \mu \mathrm{s}$, $B W=20 \mathrm{MHz}, f_{\mathrm{c}}=2 \mathrm{GHz}$, and $f_{\mathrm{d}}=1000 \mathrm{~Hz}$ arising from a relative velocity of $500 \mathrm{~km} / \mathrm{h}$ between the BS and the UEs. Letting $T_{\mathrm{c}}=1 /\left(4 f_{\mathrm{d}}\right)$, the coherence time is $T_{\mathrm{c}}=0.25 \mathrm{~ms}$. We also consider that the time elapsed between the training and the data transmission phase is equal to the coherence time of the channel, i.e., $\tau=T_{\mathrm{c}}=0.25 \mathrm{~ms}$ [17, p. 99].

Next, we compute $\sigma_{\phi}^{2}$ and $\sigma_{\varphi}^{2}$ based on a Si CMOS oscillator technology in [18]. Specifically, we consider an oscillator, whose offset level at $100 \mathrm{KHz}$ is $-105 \mathrm{dBc} / \mathrm{Hz}$. This renders $\sigma_{\phi}^{2}=$ $\sigma_{\varphi}^{2}=6.3 \times 10^{-7} \mathrm{rad}^{2}$, or $\sigma_{\phi}=\sigma_{\varphi}=0.06^{\circ}$ using [15, Eq. (4)], implying that high-quality oscillators are used at the BS and the UE. In Fig. 3, we plot the performance of the RZF precoder versus $M_{\mathrm{osc}}$ for $\tau=T_{\mathrm{c}}=0.25 \mathrm{~ms}\left(10^{4}\right.$ symbol periods $), q_{0}=$ $0.9, \beta=2$, and $M=50$ for different SNR values. At SNR = $20 \mathrm{~dB}$, we observe that the performance of the RZF precoder decreases by around 0.3 bits per channel use (bpcu), as $M_{\text {osc }}$ increases, and for $M_{\mathrm{osc}}>10$ oscillators at the BS, the additional degradation in performance becomes negligible. Furthermore, at $\mathrm{SNR}=0 \mathrm{~dB}$, the performance degradation for the RZF precoder is negligible as $M_{\mathrm{osc}}$ is increased from 1 to $M$.

\section{Rate Comparisons for CO and DO Setups}

We compare the performance of the CO setup with that of the DO setup by computing the achievable rate. Specifically, we set $q_{0}=0.9, \sigma_{\phi}=\sigma_{\varphi}=6^{\circ}$, and $\tau=K=25$, and analyze the rate performance of the RZF precoder for different values of $\beta$ in Fig. 4. For $\mathrm{SNR}=40 \mathrm{~dB}$, the performance of the $\mathrm{CO}$ setup is consistently better than that of the DO setup as the SINR used in (18) and (19) is large, and exclusively determines the achievable rate. In particular, when $\beta$ is small, the rate in (18) is a tighter upper bound, and the achievable rate is determined by the SINR term, which is relatively larger for the $\mathrm{CO}$ setup. As $\beta$ increases, the rate in (19) becomes a tighter upper bound, and the SINR term is much larger than the differential entropy term. Hence, the CO setup still performs better. Now, consider the low SNR scenario 


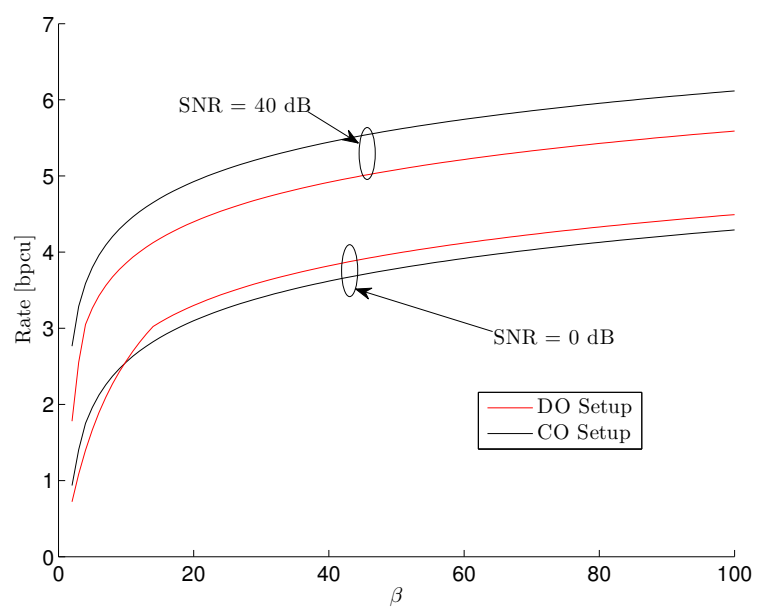

Fig. 4: $C\left(\mathrm{SINR}_{k}\right)=\min \{$ Rate in (18), Rate in (19) $\}$ of the optimized RZF precoder for the $\mathrm{CO}$ and DO setups, where $q_{0}=0.9, \sigma_{\phi}=\sigma_{\varphi}=6^{\circ}$, and $\tau=K=25$.

$(\mathrm{SNR}=0 \mathrm{~dB})$. Here, once again, for small $\beta$, the rate in $(18)$ is a tighter upper bound than that in (19), and depends on the SINR alone. Therefore, the $\mathrm{CO}$ setup performs better. However, as $\beta$ increases, the rate in (19) becomes a tighter upper bound, and the differential entropy term becomes significant compared to the SINR term. In particular, the DO setup has a lower differential entropy rate as it is only impaired by the phase noise at the UE. Consequently, the DO setup performs better.

\section{CONCLUSiOnS}

In this work, we derived the effective SINR of the RZF precoder for the GO setup in the presence of phase noise. We showed that the effect of phase noise on the SINR can be expressed as an effective reduction in the CSI quality available at the BS. Importantly, the SINR degrades as the number of oscillators, $M_{\mathrm{osc}}$, increases. This is because as $M_{\mathrm{osc}}$ increases, the desired signal power decreases, and the interference power increases. Furthermore, we showed that the variance of the random phase variations caused by the BS oscillators reduces with increasing $M_{\mathrm{osc}}$. We demonstrated that the SINR obtained is tight and agrees with that obtained from simulations with remarkable accuracy for interesting, and practical values of $M$ and $K$. Finally, we observed that for the RZF precoder, the CO setup has a higher achievable rate than the DO setup when $\beta$ is small, while the DO setup outperforms the CO setup when the $\mathrm{SNR}$ at the UE is low and $\beta$ is large.

\section{REFERENCES}

[1] F. Rusek, D. Persson, K. L. Buon, E. G. Larsson, T. L. Marzetta, O. Edfors, and F. Tufvesson, "Scaling up MIMO: opportunities and challenges with very large arrays," IEEE Signal Process. Mag., vol. 30, no. 1, pp. 40-60, Jan. 2013.

[2] G. Caire and S. Shamai, "On the Achievable throughput of a multiantenna Gaussian broadcast channel," IEEE Trans. Inf. Theory, vol. 49, no. 7, pp. 1691-1706, Jul. 2003

[3] T. Yoo and A. Goldsmith, "On the optimality of multiantenna broadcast scheduling using zero-forcing beamforming," IEEE J. Sel. Areas Commun., vol. 24, no. 3, pp. 528-541, Mar. 2006.

[4] S. Wagner, R. Couillet, M. Debbah, and D. T. M. Slock, "Large system analysis of linear precoding in correlated MISO broadcast channels under limited feedback," IEEE Trans. Inf. Theory, vol. 58, no. 7, pp. 4509-4537, Jul. 2012.
[5] A. Müller, A. Kammoun, E. Bjornson, and M. Debbah, "Linear precoding based on truncated polynomial expansion - part I: large-scale single-cell systems," IEEE J. Sel. Topics Signal Process., vol. 8, no. 5, pp. 815-830, Oct. 2014.

[6] E. Bjornson, J. Hoydis, M. Kountouris, and M. Debbah, "Massive MIMO systems with non-ideal hardware: energy efficiency, estimation, and capacity limits," IEEE Trans. Inf. Theory, vol. 60, no. 11, pp. 7112-7139, Nov. 2014.

[7] R. Krishnan, M. Reza Khanzadi, N. Krishnan, A. Graell i Amat, T. Eriksson, N. Mazzali, and G. Colavolpe, "On the impact of oscillator phase noise on the uplink performance in a massive MIMO-OFDM system," IEEE Signal Process. Lett., 2014, submitted. [Online]. Available: http://arxiv.org/abs/1405.0669.

[8] A. Pitarokoilis, S. K. Mohammed, and E. G. Larsson, "Uplink performance of time-reversal MRC in massive MIMO system subject to phase noise," IEEE Trans. Wireless Commun., accepted for publication. [Online]. Available: http://arxiv.org/abs/1306.4495

[9] K. T. Truong and R. W. Heath Jr., "Effects of channel aging in massive MIMO systems," IEEE J. Commun. Netw. (Special Issue on Massive MIMO), vol. 15, no. 4, pp. 338-351, Aug. 2013.

[10] R. Krishnan, A. Graell i Amat, T. Eriksson, and G. Colavolpe, "Algorithms for Joint Phase Estimation and Decoding for MIMO Systems in the Presence of Phase Noise and Quasi-static Fading Channels," accepted for publication in IEEE Trans. Signal Process., 2015. [Online]. Available: http://arxiv.org/abs/1312.2232.

[11] Q. Zhang, S. Jin, K.-K. Wong, H. Zhu, and M. Matthaiou, "Power scaling of uplink massive MIMO systems with arbitrary-rank channel means," IEEE J. Sel. Topics Signal Process., vol. 8, no. 5, pp. 966-981, Oct. 2014.

[12] B. Makki and T. Eriksson, "Feedback subsampling in temporally-correlated slowly-fading channels using quantized CSI," IEEE Trans. Commun., vol. 61, no. 6, pp. 2282-2294, Jun. 2013.

[13] A. Lapidoth, "On phase noise channels at high SNR," in Proc. IEEE Inf. Theory Workshop (ITW), Bangalore, India, Oct. 2002, pp. 14.

[14] A. M. Tulino and S. Verdu, Random matrix theory and wireless communications. Delft, Netherlands: Now Publishers Inc., 2004.

[15] R. Krishnan, M. R. Khanzadi, N. Krishnan, Y. Wu, A. Graell i Amat, T. Eriksson, and R. Schober, "Linear Massive MIMO Precoders in the Presence of Phase Noise - A Large-Scale Analysis," IEEE Trans. Veh. Tech., under review. [Online]. Available: http://arxiv.org/abs/1501.05461

[16] M. Reza Khanzadi, G. Durisi, and T. Eriksson, "Capacity of multiple-antenna phase-noise channels with common/separate oscillators," IEEE Trans. Commun., Sep. 2014, submitted. [Online]. Available: http://arxiv.org/abs/1409.0561.

[17] S. Sesia, I. Toufik, and M. Baker, Eds., LTE: The UMTS long term evolution. John Wiley and Sons, 2009.

[18] Hittite Microwave Corporation, "Ku-Band MMIC VCO with divide-by-8, 14.0 - 15.0 GHz," HMC398QS16G datasheet, Feb. 2013. 